\title{
Correction to: Alpha-1 Antitrypsin Deficiency: a Rare Disease?
}

\section{Roxana Cortes-Lopez ${ }^{1} \cdot$ Igor Barjaktarevic $^{1}$}

Published online: 12 September 2020

(C) Springer Science+Business Media, LLC, part of Springer Nature 2020

\section{Correction to: Current Allergy Asthma Reports (2020) 20:51 https://doi.org/10.1007/s11882-020-00942-4}

The original version of this article contained errors in Table 3 on page 6. In the last row, Trypsone was listed as a Grifols product in EU. This product has been discontinued.

The corrected table is presented in this erratum.

Table 3 Available augmentation replacement therapies for alpha-1 antitrypsin deficiency

\begin{tabular}{|c|c|c|c|c|c|c|c|c|}
\hline Product & Manufacturer & Country & Formulation & Dose & $\begin{array}{l}\text { Dosing } \\
\text { interval }\end{array}$ & $\begin{array}{l}\text { Concentration } \\
\text { after } \\
\text { reconsitution }\end{array}$ & Rate of infusion & $\begin{array}{l}\text { Duration } \\
\text { of infusion }\end{array}$ \\
\hline Prolastin C & Grifols & USA & Lyophylized & $60 \mathrm{mg} / \mathrm{Kg}$ & Weekly & $50 \mathrm{mg} / \mathrm{mL}$ & $0.08 \mathrm{ml} / \mathrm{kg} / \mathrm{min}$ & $15 \mathrm{~min}$ \\
\hline Prolastin C Liquid & Grifols & USA & Liquid & $60 \mathrm{mg} / \mathrm{Kg}$ & Weekly & $50 \mathrm{mg} / \mathrm{mL}$ & $0.08 \mathrm{ml} / \mathrm{kg} / \mathrm{min}$ & $15 \mathrm{~min}$ \\
\hline Glassia & Takeda/Kamada & USA/Israel & Liquid & $60 \mathrm{mg} / \mathrm{Kg}$ & Weekly & $20 \mathrm{mg} / \mathrm{mL}$ & $0.04 \mathrm{~mL} / \mathrm{kg} / \mathrm{min}$ & $60-80 \mathrm{~min}$ \\
\hline Aralast & Takeda & USA & Lyophylized & $60 \mathrm{mg} / \mathrm{Kg}$ & Weekly & $20 \mathrm{mg} / \mathrm{mL}$ & $0.08 \mathrm{ml} / \mathrm{kg} / \mathrm{min}$ & $40 \mathrm{~min}$ \\
\hline Zemaira & CSL Behring & USA & Lyophylized & $60 \mathrm{mg} / \mathrm{Kg}$ & Weekly & $50 \mathrm{mg} / \mathrm{mL}$ & $0.08 \mathrm{ml} / \mathrm{kg} / \mathrm{min}$ & $15 \mathrm{~min}$ \\
\hline Alfalastin & $\begin{array}{l}\text { Laboratoire français } \\
\text { fractionnement et de } \\
\text { Biotechnologies }\end{array}$ & Frace & Lyophylized & $60 \mathrm{mg} / \mathrm{Kg}$ & Weekly & $50 \mathrm{mg} / \mathrm{mL}$ & $4 \mathrm{ml} / \mathrm{mn}$ & $15 \mathrm{~min}$ \\
\hline Respreeza & CSL Behring & $\mathrm{EU}$ & Lyophylized & $60 \mathrm{mg} / \mathrm{Kg}$ & Weekly & $50 \mathrm{mg} / \mathrm{mL}$ & $0.08 \mathrm{ml} / \mathrm{kg} / \mathrm{min}$ & $15 \mathrm{~min}$ \\
\hline Prolastin/Prolastina & Grifols & $\mathrm{EU}$ & Lyophylized & $60 \mathrm{mg} / \mathrm{Kg}$ & Weekly & $25 \mathrm{mg} / \mathrm{mL}$ & $0.08 \mathrm{ml} / \mathrm{kg} / \mathrm{min}$ & $30 \mathrm{~min}$ \\
\hline
\end{tabular}

Publisher's Note Springer Nature remains neutral with regard to jurisdictional claims in published maps and institutional affiliations.

The online version of the original article can be found at https://doi.org/ $10.1007 / \mathrm{s} 11882-020-00942-4$

Igor Barjaktarevic ibarjaktarevic@mednet.ucla.edu

1 Division of Pulmonary and Critical Care Medicine, David Geffen School of Medicine at UCLA, 10833 Le Conte Avenue, CHS, Los Angeles, CA 90095, USA 